\title{
DISTRIBUIÇÃO GEOGRÁFICA DAS CHUVAS NO ESPAÇO URBANO DE CATALÃO EM 2015-2016 (GO)
}

\author{
Thiago Patrocínio da Silva ${ }^{(1)}$,Ayr Carvalho Costa ${ }^{(2)}$ Rafael de Ávila Rodrigues ${ }^{(3)}$ Silas Pereira \\ Trinidade $^{(4)}$
}

(1) Unidade Acadêmica Especial Instituto de Geografia/Universidade Federal de Goiás/Regional Catalão, thiago.patrocinio@gmail.com

(2) Unidade Acadêmica Especial Instituto de Geografia/ Universidade Federal de Goiás/Regional Catalão, istrovado@ hotmail.com

(3) Unidade Acadêmica Especial Instituto de Geografia/Universidade Federal de Goiás/Regional Catalão, Rafael.avila.roodrigues@gmail.com

(4) Unidade Acadêmica Especial Instituto de Geografia/Universidade Federal de Goiás/ Regional Catalão, silaspereiratrinidade@gmail.com

\section{Eixo 3: Climatologia em diferentes níveis escalares: mudanças e variabilidades}

\section{Resumo}

\begin{abstract}
O presente trabalho objetivou analisar a distribuição das chuvas no espaço urbano de Catalão (GO) através de um total quantitativo em diferentes bairros da cidade compreendido entre setembro de 2015 a março de 2016. Os dados apresentados foram coletados através de uma rede de pluviômetros, modelo Ville de Paris, instalados em vinte pontos estratégicos da cidade, a 1,5m do solo. Observou-se através do mapa de isoietas que o local mais chuvoso é a estação ETA DIMIC com uma média de 1366,6 a $1440 \mathrm{~mm}$ e com menos incidência é a estação Escola CAIC tendo uma média de apenas 890,9 a 973,1mm.
\end{abstract}

Palavras chave: rede pluviométrica, Catalão, distribuição, precipitação.

\section{Introdução}

O estudo do tempo e do clima ocupa uma importante posição no amplo campo da ciência ambiental. Processos atmosféricos tem a capacidade de influenciar os processos em outras partes do ecossistema, especialmente na biosfera, hidrosfera e litosfera. Semelhantemente, os processos e as outras partes do ambiente não podem ser ignorados pelos estudiosos do tempo e do clima (AYOADE, 1996).

Precipitação, segundo a meteorologia, é qualquer depósito em forma líquida ou sólida derivado da atmosfera, como por exemplo: chuva, granizo, neblina, orvalho dentre outros. Pode-se considerar a precipitação em três tipos básicos, tendo como base o estilo de elevação do ar que tenha dado origem à mesma. São eles: tipo convectivo, que é conexo à instabilidade convectiva, tipo ciclônico, que é associado com convergência em uma depressão, e pôr fim a orográfica que é associada às áreas irregulares ou montanhosas. A precipitação pode ser medida com um instrumental denominado de pluviômetro, normalmente, expressa em milímetros (AYOADE, 1996). 
XVII Simpósio Brasileiro

de Geografia Fisica Aplicada

I Congresso Nacional de Geografia Física
OS DESAFIOS DA GEOGRAFIA FÍSICA NA FRONTEIRA DO CONHECIMENTO

Instituto de Geociências - Unicamp

Campinas - SP

28 de Junho à 02 de Julho de 2017

O Estado de Goiás é caracterizado por um período chuvoso (outubro a abril) e outro seco (maio a setembro). "No período chuvoso ocorre $95 \%$ do total de precipitação pluvial com destaque para os meses de meses de dezembro e janeiro, que mostram que na maior parte do estado chove em torno de 250 a 300 mm" (GOIÁS, ESTADO, 2006).

As características atmosféricas de um determinado local são influenciadas pelas condições reinantes no lugar resultante da combinação de algumas grandezas físicas denominadas elementos climáticos. Tais condições são chamadas de tempo meteorológico, popularmente chamado de condições do tempo. "O clima seria a síntese, a generalização das diferentes condições de tempo prevalecentes nesse lugar, e considera um número bem maior de dados, com a frequência de ocorrências de alguns fenômenos meteorológicos mais comuns no local, além das condições médias de tempo" (AYOADE, 2002).

O clima goiano é predominantemente tropical, com a divisão marcante de duas estações bem definidas durante o ano: verão úmido, nos meses de dezembro a março, e inverno seco, predominante no período de junho a agosto. De acordo com o Sistema de Meteorologia e Hidrologia da Secretaria de Ciência e Tecnologia (SIMEHGO/SECTEC), a temperatura média oscila entre $18^{\circ} \mathrm{C}$ e $26^{\circ} \mathrm{C}$, com amplitude térmica significativa, variando segundo o regime dominante no Planalto Central.

A visualização das conexões atmosféricas depende das respostas locais colhidas nas variações diárias e horárias dos elementos do clima (medições em superfície: estações e postos meteorológicos), nas cartas sinóticas do tempo e nas imagens fornecidas por satélites meteorológicos. Interpretar e conjugar toda essa gama de informações e, apartir daí, vislumbrar o ritmo de sucessão das massas de ar e dos tipos de tempo, isto é, o próprio movimento da atmosfera, é uma ação de abordagem eminentemente geográfica, ainda que se esteja a lidar com informações predominantemente meteorológicas (ZAVATINI, 2004).

Tais informações como da quantificação diária, mensal e anual de precipitação irão ajudar no dimensionamento de projetos de irrigação, construções de barragens, melhoramento do planejamento de sistemas de manejos culturais, além do uso eficiente do solo e dos recursos hídricos. Assim, o objetivo do presente trabalho foi analisar a distribuição das chuvas no espaço urbano de Catalão no período de setembro de 2015 a março de 2016, cujo período compreende a estação chuvosa na área de estudo. 


\section{2. Metodologia de Pesquisa}

\section{1 Área de Estudo}

Catalão é um municípiobrasileiro do estado de Goiás. Localiza-se à latitude $18^{\circ} 9^{\prime} 57^{\prime \prime}$ sul e à longitude $47^{\circ}$ 56' 47" oeste, e à altitude de 835 metros, conforme exposto na figura 01. Sua população, de acordo com estimativas do Instituto Brasileiro de Geografia e Estatística (IBGE, 2015), é de 98.737 habitantes.

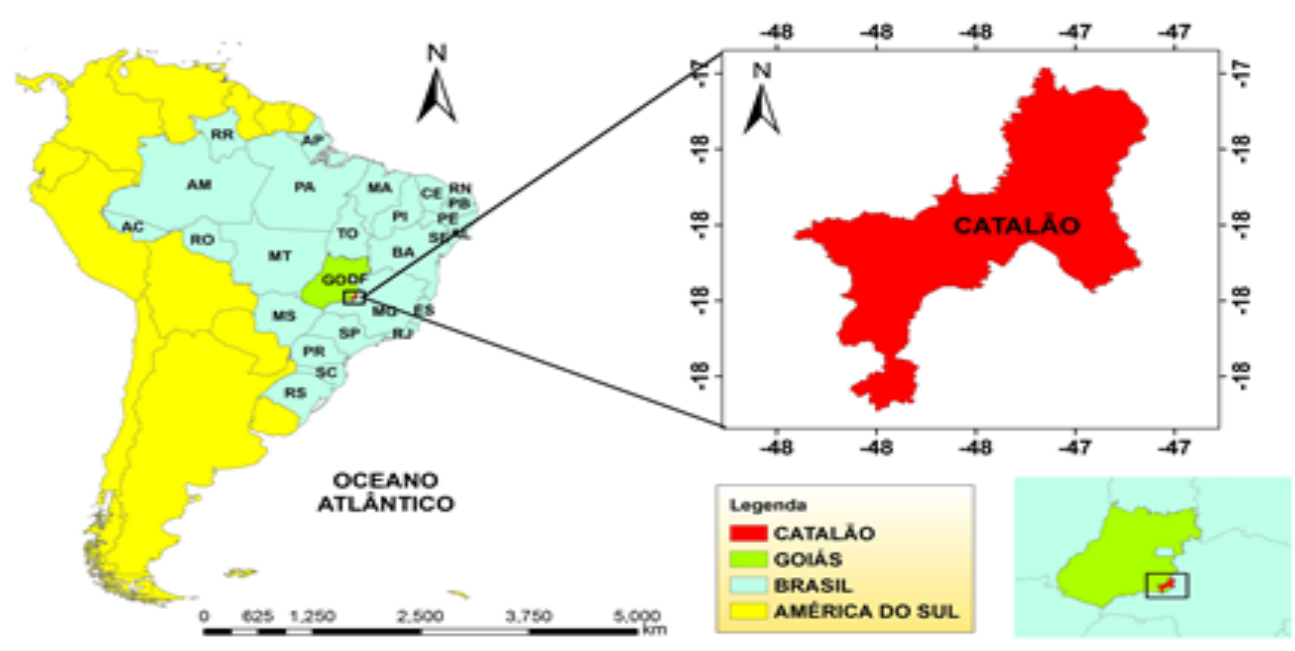

Figura 01- Localização geográfica da área de estudo e recorte do município de Catalão (GO) Fonte: IBGE (2004).

A precipitação é uma variável que proporciona uma grande variabilidade temporal, sobretudo, na escala de tempo interanual. A figura 02 ilustra a distribuição dos totais mensais de chuva no município de Catalão, no período de 1961-1990 de acordo com o Instituto Nacional de Meteorologia (INMET).

Assim sendo, pôde-se observar que as menores ocorrências de chuvas no município foram registradas nos meses de junho e julho, historicamente, com apenas 10,0 e 10,3 mm respectivamente, o que caracteriza os períodos de estiagem crítica para a região, ao passo que os maiores valores registrados ocorreram em dezembro, apresentando o total de $283,5 \mathrm{~mm}$, caracterizando o período de chuvas da região, o que ressalta uma alta variabilidade, a qual pode ser observada nesses períodos secos e chuvosos. Destaca-se para precipitação anual de $1484,8 \mathrm{~mm}$. Mais detalhes sobre a caracterização climática na região Sudeste de Goiás podem ser encontrados em Rodrigues et al. (2009 e 2012). 


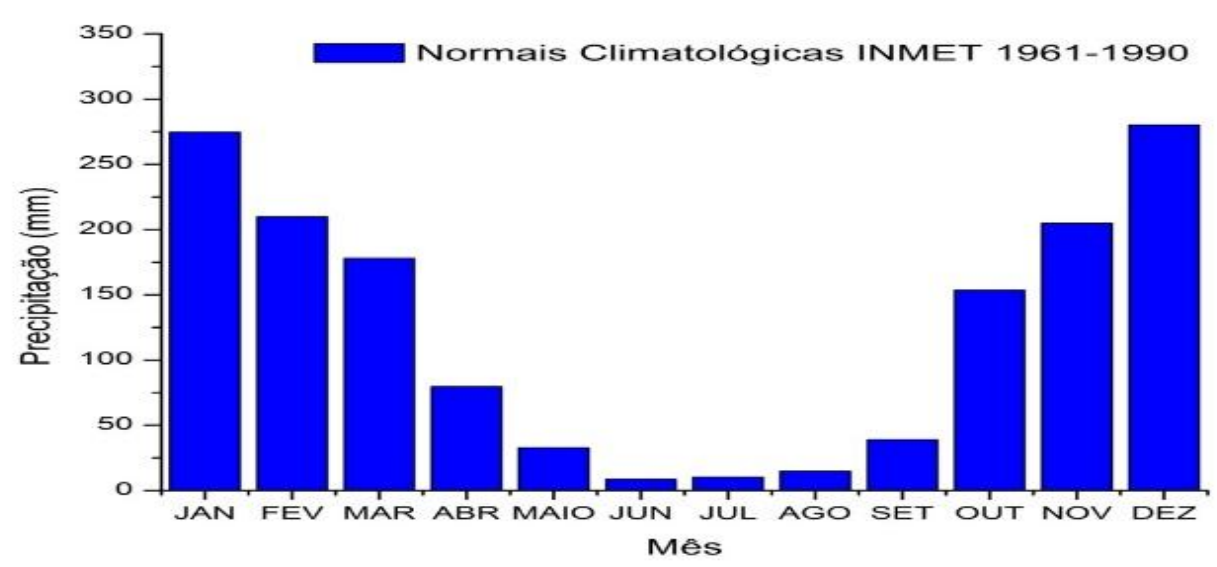

Figura 02 - Precipitação Pluviométrica em Catalão (GO) de 1961-1990 Fonte - INMET - Normais Climatológicas 1961-1990.

\subsection{Instalação e monitoramento da rede pluviométrica}

A rede pluviométrica no espaço urbano da cidade de Catalão - GO (figura 03) teve como objetivo principal acompanhar as distribuições dos totais pluviométricos responsáveis para o mapeamento e correlação da estrutura espacial das chuvas na cidade.

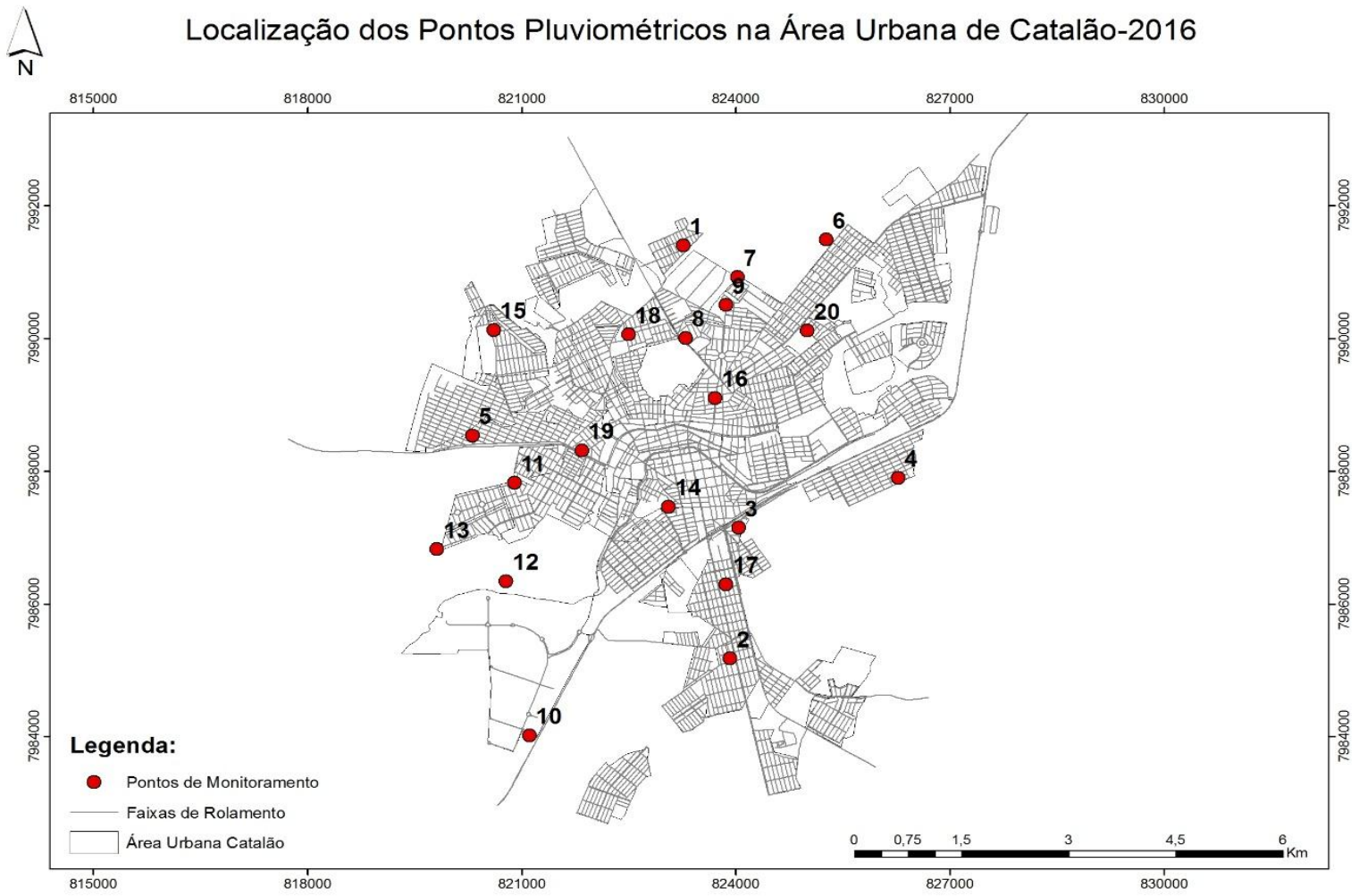

Figura 03: Localização geográfica dos pluviômetros no espaço urbano de Catalão (GO) Fonte: Adaptado pelo autor 


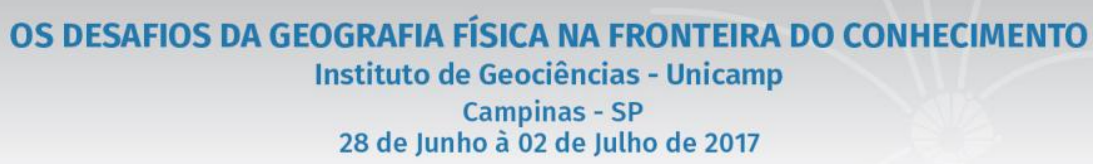

Diante disso, foram instalados 20 (vinte) pluviômetros do tipo Ville de Paris (figura 03), a 1,5m acima do solo, conforme orientações do INMET/CPTEC, de forma equitativa no espaço urbano para a coleta dos dados pluviométricos.

Os pluviômetros foram adquiridos por meio de parceria entre a Universidade Federal de Goiás - Regional Catalão - Unidade Acadêmica Especial Instituto de Geografia e a Superintendência de Água e Esgoto (SAE) do referido Município.

As leituras dos pluviômetros foram realizadas por meio de coletas diárias praticadas pelos responsáveis por cada localidade, sendo que para estas pessoas foi fornecido um treinamento de como proceder na medida da precipitação.

As leituras foram realizadas todos os dias às $09 \mathrm{~h} 00$, sendo registradas em mm de chuva a cada 24 horas.

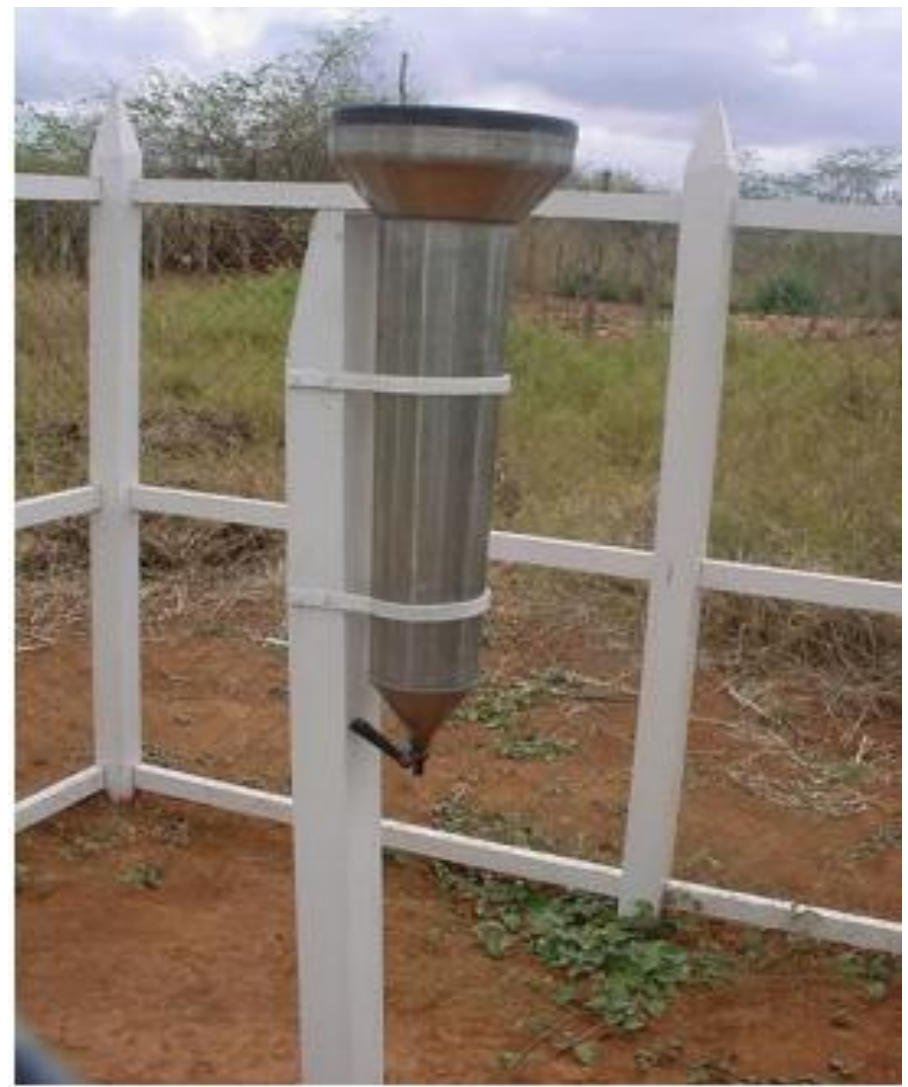

Figura 04: Pluviômetro tipo Ville de Paris

Fonte: JCTM Comércio e Tecnologia - Fabricante do pluviômetro.

Para a escolha dos locais da instalação dos pluviômetros foi considerado a acessibilidade do local para a coleta de dados pluviométricos, ausência de árvores, prédios casas, muros, etc. conforme mostra a Tabela I. 


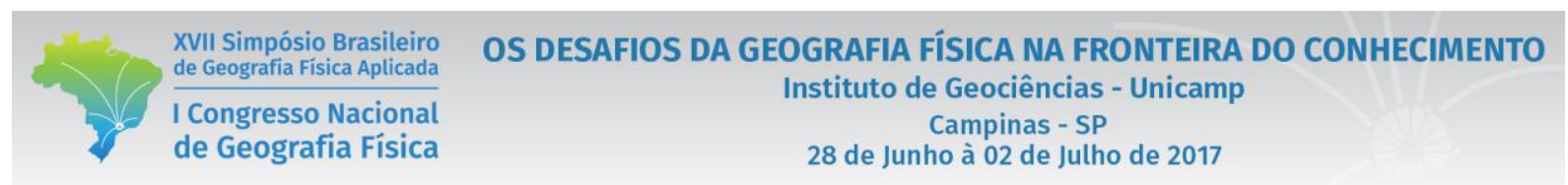

\begin{tabular}{|c|c|c|c|c|}
\hline PLUVIÔMETRO & ENDEREÇO & \multicolumn{2}{|c|}{ COORDENADAS } & ALTITUDE \\
\hline P 01 & $\begin{array}{c}\text { Evelina Nour II - Escola } \\
\text { Municipal }\end{array}$ & $18^{\circ} 08^{\prime} 31.74^{\prime \prime S}$ & $47^{\circ} 56^{\prime} 42.42^{\prime \prime O}$ & 889 \\
\hline P 02 & Estrela - Reservatório - 500m & $18^{\circ} 11 ' 53.54^{\prime \prime S}$ & $47^{\circ} 56 ' 16.41^{\prime \prime O}$ & 871 \\
\hline P 03 & $\begin{array}{l}\text { Castelo Branco II - } \\
\text { Reservatórios 500m³ }\end{array}$ & $19^{\circ} 10^{\prime} 49.53^{\prime \prime S}$ & $47^{\circ} 56 ' 13.54^{\prime \prime O}$ & 888 \\
\hline P 04 & $\begin{array}{c}\text { Pontal Norte - Reservatório } \\
500 \mathrm{~m}^{3}\end{array}$ & $18^{\circ} 10^{\prime} 23.72^{\prime \prime S}$ & $47^{\circ} 54^{\prime} 57.88^{\prime \prime O}$ & 897 \\
\hline P 05 & $\begin{array}{l}\text { Escola Municipal Wilson da } \\
\text { Paixão }\end{array}$ & $18^{\circ} 10^{\prime} 06.26^{\prime \prime S}$ & $47^{\circ} 58 ' 21.00^{\prime \prime O}$ & 866 \\
\hline P 06 & ETA Ipanema & $18^{\circ} 8^{\prime} 27.80^{\prime \prime S}$ & $47^{\circ} 55 ' 34.34^{\prime \prime O}$ & 907 \\
\hline P 07 & ElevatóriaLeblon & $18^{\circ} 8^{\prime} 46.84^{\prime \prime} \mathrm{S}$ & $47^{\circ} 56 ' 16.24^{\prime \prime O}$ & 886 \\
\hline P 08 & ETA & $18^{\circ} 9$ '16.97"S & $47^{\circ} 56^{\prime} 40.29^{\prime \prime O}$ & 930 \\
\hline P 09 & CondomínioResidencialOlinda & $18^{\circ} 09^{\prime} 00.52^{\prime \prime} \mathrm{S}$ & $47^{\circ} 56 ' 21.59^{\prime \prime O}$ & 910 \\
\hline P 10 & ETA - Dimic & $18^{\circ} 12 ' 32.75^{\prime \prime S}$ & $47^{\circ} 57^{\prime} 51.51^{\prime \prime O}$ & 852 \\
\hline P 11 & $\begin{array}{l}\text { Alto da Boa Vista - } \\
\text { Reservatório }\end{array}$ & $18^{\circ} 10^{\prime} 29.20^{\prime \prime S}$ & $47^{\circ} 5810.76^{\prime \prime O}$ & 866 \\
\hline P 12 & ETE - Dimic & 18'11'17.36"S & $47^{\circ} 58 ' 3.83$ "O & 816 \\
\hline P 13 & $\begin{array}{l}\text { Escola Municipal } \\
\text { StaTerezinha }\end{array}$ & $18^{\circ} 11^{\prime} 02.30^{\prime \prime S}$ & $47^{\circ} 58^{\prime} 37.10^{\prime \prime O}$ & 862 \\
\hline P 14 & JK - LabibeFayad & $18^{\circ} 10^{\prime} 39.66^{\prime \prime S}$ & $47^{\circ} 56^{\prime} 47.14^{\prime \prime O}$ & 900 \\
\hline P 15 & BairroPaineiras - Rua 2006 & $18^{\circ} 09^{\prime} 14.50 " \mathrm{~S}$ & $47^{\circ} 58^{\prime} 11.95^{\prime \prime O}$ & 886 \\
\hline P 16 & Setor Paraíso & $18^{\circ} 09^{\prime} 46.14^{\prime \prime S}$ & $47^{\circ} 56^{\prime} 25.88^{\prime \prime O}$ & 854 \\
\hline P 17 & Rodovia GO 330 & 18'11'17.29"S & $47^{\circ} 56 ' 19.11^{\prime \prime O}$ & 867 \\
\hline P 18 & Escola CAIC & $18^{\circ} 09^{\prime} 15.61^{\prime \prime S}$ & $4^{\circ} 57^{\prime} 07.70^{\prime \prime O}$ & 903 \\
\hline P 19 & Poço 20 Agosto - Último & $18^{\circ} 10^{\prime} 48.82^{\prime \prime} S$ & $47^{\circ} 57^{\prime} 23.17^{\prime \prime O}$ & 819 \\
\hline P 20 & Clube do Povo & $18^{\circ} 10^{\prime} 7.10^{\prime \prime} \mathrm{S}$ & $47^{\circ} 58^{\prime} 15.28^{\prime \prime O}$ & 859 \\
\hline P 21 & PIO GOMES & $18^{\circ} 10^{\prime} 12.77^{\prime \prime S}$ & $47^{\circ} 57^{\prime} 28.92^{\prime \prime O}$ & 859 \\
\hline P 22 & UFG/RC & 1809'12.43"S & $47^{\circ} 55^{\prime} 42.47^{\prime \prime O}$ & 906 \\
\hline
\end{tabular}

Tabela I. Localização dos pontos onde os pluviômetros foram instalados no espaço urbano de Catalão (GO). Fonte: Elaborada pelo autor.

\section{Resultados}

Foi possível observar através do mapa de isoietas que não existe uma homogeneidade na distribuição das chuvas no espaço urbano sendo que os dados de chuva obtidos variam de 890,9mm a $1440 \mathrm{~mm}$.

Dentre os vinte e dois pontos instalados, os que se encontram localizados nas áreas mais adensadas da cidade, e com altitudes mais elevadas, obtiveram uma quantidade menor de chuva em relação aos que se encontram em partes com menos ocupações e com altitudes mais baixas, conforme pode ser observado na figura 05 .

DOI - 10.20396/sbgfa.v1i2017.2426 - ISBN 978-85-85369-16-3 


\section{Isoietas da Precipitação Total na Área Urbana de Catalão-2015/2016}

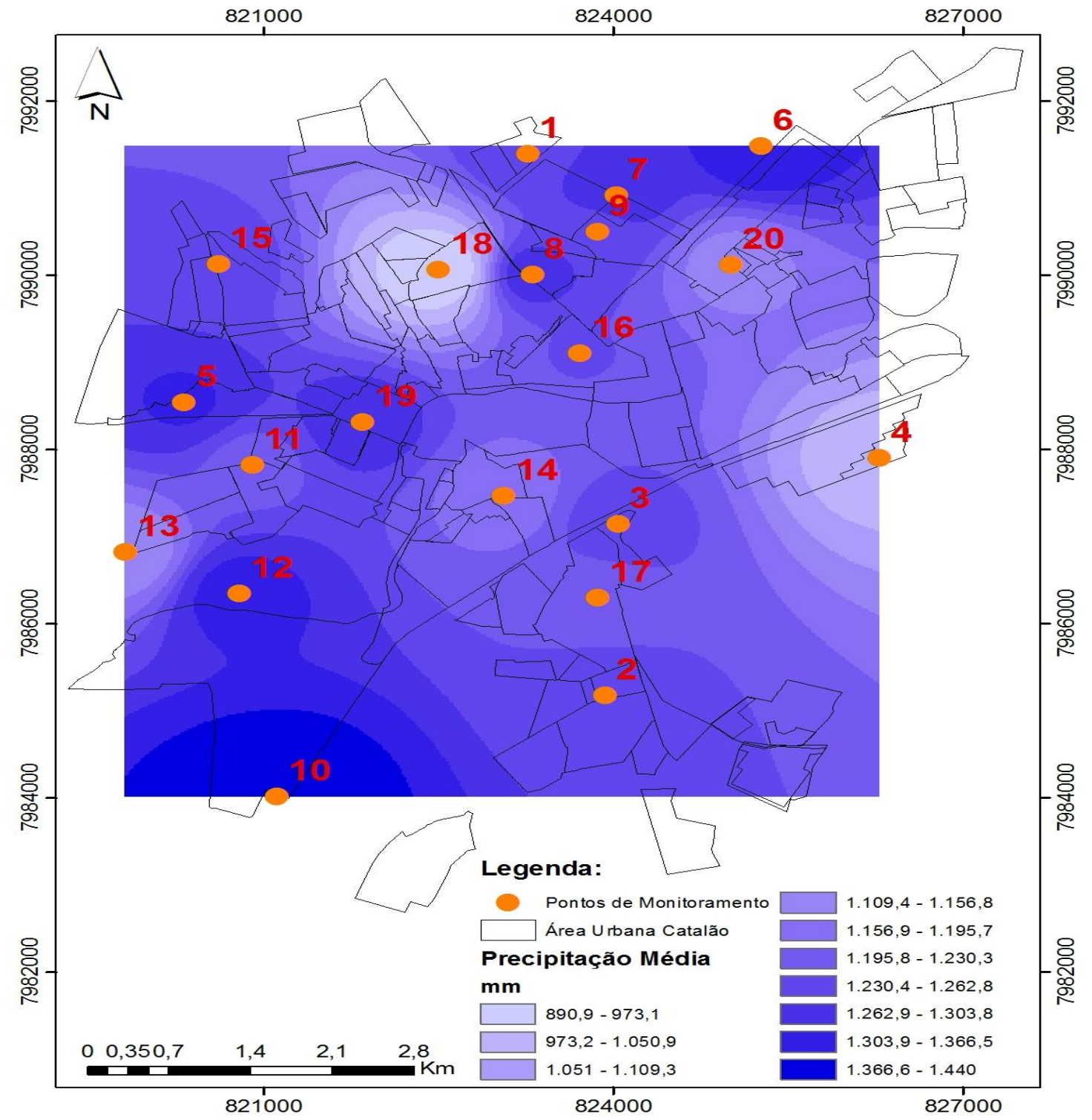

Figura 05: Mapa de Isoietas da Precipitação Total na área urbana de Catalão-2015/2016

Fonte: Adaptado pelo autor

\section{Conclusão}

Por meio dos resultados obtidos, e com as discussões desenvolvidas, chega-se à conclusão de que existe uma diversidade na distribuição da chuva na área urbana do município de Catalão-GO. Ou seja, não existe uma homogeneidade na precipitação, que pode ser ocasionada pela diferença de altitude. 
Assim, por meio do mapa de isoietas, figura 05, foi possível observar as áreas que recebem maiores volumes de água, com destaque para a porção sudoeste da cidade por ser a região mais chuvosa; local onde se encontra situado o ponto 10 (P-10), denominado de "ETA DIMIC", com uma média de 1366,6 a $1440 \mathrm{~mm}$. Assim, é possível destacar que os pontos localizados às margens do Vale do Pirapitinga foram os pontos com maiores volumes precipitados.

Outro ponto da cidade que merece atenção é a região centro-norte que, como foi demostrado na figura 05 , teve a menor incidência pluviométrica. Esse setor é onde se encontra situado o ponto 18 (P-18), intitulado como "Escola CAIC", tendo uma média de apenas 890,9 a 973,1mm, dessa forma, conclui-se que as áreas de menor adensamento populacional, área essas ainda com baixo índice de ação antropomórfica foram as áreas onde registrou-se os menores índices pluviométricos.

As demais regiões monitoradas descritas no mapa apresentaram uma média regular, bem como mais similar em relação à distribuição das chuvas, não apresentando resultados de destaque. As médias de precipitação das outras regiões, ou seja, nordeste, noroeste, sudeste e centro-oeste tiveram como valores de mínimas de chuva 1156,9 a $1195,7 \mathrm{~mm}$ e máximas de chuva 1303,9 a $1366,5 \mathrm{~mm}$.

\section{Referências}

AYOADE, J. Introdução à Climatologia para os Trópicos. Trad. Maria JuracoZani dos Santos. 3.ed. Rio de Janeiro: Bertrand Brasil, 1991.

ALVES MAZZOTTI, A. J. Revisão da Bibliografia. In: ALVES MAZZOTTI, A. J. \& GEWANDSZNAJDER, F. O Método nas Ciências Naturais e Sociais: pesquisa quantitativa e qualitativa. São Paulo: Pioneira, 2002. P. 179-188. Cap. 8.

DEMO, Pedro. Metodologia do conhecimento científico. São Paulo: Atlas, 2000.

GOIÁS (Estado). Secretaria de Indústria e Comércio. Superintendência de Geologia e Mineração. Caracterização Climática do Estado de Goiás. Por Silvando Carlos da Silva, Neiva Maria Pio de Santana, José Cardoso Pelegrini. Goiânia, 2006. 133 p. il. (Série Geologia e Mineração n. 3).

INSTITUTO NACIONAL DE METEOROLOGIA (INMET). Mapas de Condições Registradas. Disponível em: http://www.inmet.gov.br/mapa_condicoes_encontradas. Acesso em 08 de janeiro de 2015.

LUNA, S. V. Planejamento de pesquisa: uma introdução. São Paulo: EDUC, 2005. (Série Trilhas).

NIMER, E. In:Revista Brasileira' de Geografia 1971 e 1972. Climatologia da Região Centro-Oeste do Brasil. Introdução à Climatologia Dinâmica. Rio de Janeiro, IBG Fundação IBGE. P.3- 33, 1971 e 1972.

RODRIGUES, R. A.; OLIVEIRA, G. A.; FARIA, A. L. L.; OLIVEIRA JÚNIOR. Caracterização Climática no entorno da Usina Hidroelétrica Serra do Facão (GO). In: TORRES, F. T. P.; DAGNINO, R. S.; OLIVEIRA JÚNIOR, A. (Orgs.). Contribuições Geográficas, p. 521-542, 2009.

RODRIGUES, R. A.; OLIVEIRA, G. A.; FARIA, A. L. L.; OLIVEIRA JÚNIOR, A.; DELGADO, R. C. Determinação de Regiões climaticamente homogêneas no Estado de Goiás. In: ASSIS, A. A. F.; FARIA, A. L. L (Orgs.). O onde e o quando: espaço e memória na construção da história e da geografia, p. 274-289, 2012.

SISTEMA DE METEOROLOGIA E HIDROLOGIA DO ESTADO DE GOIÁS, Disponível em: http://www.simehgo.sectec.go.gov.br. Acesso em 30 de julho de 2016. 
SORRE, M. (1934). Objectandmetodofclimatology. Revista do Departamento de Geografia, n. 18, p. 89-94.

ZAVATINI, João Afonso. Estudos do Clima no Brasil. Campinas, SP: Editora Alínea, 2004. 398 p.

ZAMBOTTI, J. L. (2001). Mapas pluviométricos médios mensais no verão para o Estado do Paraná, utilizando métodos geoestatisticos. Botucatu, 117 p. Tese (Doutorado em Agronomia) - Universidade Estadual Paulista "Júlio de Mesquita Filho", Faculdade de Ciências Agronômicas Campus de Botucatu.

\section{Agradecimentos}

O autor agradece à Fundação de Amparo à Pesquisa do Estado de Goiás (FAPEG) pela concessão da bolsa de Iniciação Científica, ao CPTPI - Centro de Pesquisas Tecnológicas e Processamento de Imagem da Universidade Federal de Goiás - Regional Catalão, pela infraestrutura disponível no desenvolvimento da pesquisa, e à Superintendência de Água e Esgoto pelo financiamento dos pluviômetros. 